\title{
Ampliación del rango geográfico del Escorpión de Agua Curicta scorpio Stål, (Hemiptera: Nepidae) y primer registro para Costa Rica
}

\section{Geographic range extension of the Waterscorpion Curicta scorpio Stål, (Hemiptera: Nepidae) and first record for Costa Rica}

\author{
Federico Herrera
}

Escuela de Biología, Oficina de Posgrado en Biología, Universidad de Costa Rica, 11501-2060 San Pedro, Montes de Oca, San José Costa Rica.

Email: federico.herrera@ucr.ac.cr

Citación:

Herrera F. 2013. Ampliación del rango geográfico del Escorpión de Agua Curicta scorpio Stål, (Hemiptera: Nepidae) y primer registro para Costa Rica. Rev. peru. biol. 20(2): 197 - 198 (Diciembre 2013)

\section{Resumen}

Se registra por primera vez para Costa Rica la presencia de Curicta scorpio Stål. Este registro confirma la presencia del género en Costa Rica y la ampliación de su distribución sur.

Palabras clave: nuevo registro; biodiversidad; Centro América.

\section{Abstract}

Curicta scorpio Stål.c is registered for first time to Costa Rica. With this record this genus is confirmed in Costa Rica and its southern range distribution in turn is expanded.

Keywords: first record; biodiversity; Central America.

La Familia Nepidae está representada en el continente americano por los géneros Telmatotrephes Stål, Ranatra Fabricius, Curicta Stål y Nepa Linnaeus (Štys \& Jansson 1988). Para la región centroamericana sólo se han registrado dos géneros, Curicta y Ranatra (Champion 1901). Springer (1998, 2004) reporta para Costa Rica el género Curicta pero sin especificar la especie; mientras que Ranatra cuenta con una especie reportada para el país, Ranatra pittieri Montandon (Montandon 1910, Herrera 2013).

Por otra parte, el género Curicta posee hasta el momento dos especies en Centroamérica, Curicta scorpio Stål que se encuentra distribuida desde la parte sur de los estados de Mississippi (Lago 2000) Luisiana y Texas, en Estados Unidos hasta Nicaragua y Curicta carinata Kuitert que se distribuye en Argentina, Paraguay, Bolivia, Perú, Ecuador, Colombia, Trinidad y Panamá (Keffer 1997, Mazzucconi et al. 2009b).

Se registra por primera vez para Costa Rica la presencia de C. scorpio. Para su identificación se utilizó la clave provista por Keffer (1997). Los tres especímenes hembra examinados (montados en seco) se encontraban depositados dentro de la colección entomológica de enseñanza, de la Escuela de Biología de la Universidad de Costa Rica, Sede Rodrigo Facio; este material seco se trasladó a la Colección de Entomología del Museo de Zoología de la Universidad de Costa Rica (MZUCR). Mientras que el espécimen macho (conservado en etanol 70\%) se encuentra depositado en la Colección de Entomología Acuática del MZUCR.

Material examinado: Provincia de Alajuela: San Ramón, 1000 m.s.n.m. 18-III2008. Col. E. Villegas (†). Provincia de Guanacaste: Cañas, 600 m.s.n.m. 29-II2008. Col. P. Gutiérrez (+); Bagaces, Palo Verde, en canales de arrozales, 50 m.s.n.m. 06-XI-1999. Col. M. Springer (ô); Parque Nacional Palo Verde. 20 m. 13-III-2003. Col. C. Sánchez () .

Las especies de este género se encuentran habitualmente en ambientes lénticos poco profundos o pequeños arroyos, vegetados, con fondo de barro y entre la hojarasca del fondo. Sus hembras depositan los huevos en las orillas y los cubren de barro (Menke 1979, Keffer 1997, Mazzucconi et al. 2009a). Este género realiza vuelos nocturnos 
de dispersión (Sites \& Polhemus 1994). Wiley (1924) y Keffer y colaboradores (1994) describieron los cinco estadios ninfales de C. scorpio. El proceso de cópula para esta misma especie fue descrita por Wiley (1922) y Keffer \& McPherson (1993).

Curicta scorpio es la única especie del género hasta el momento con distribución en Norte- y Centroamérica (Keffer 1997). Con estos cuatro individuos se confirma la presencia de la primera especie de éste género en Costa Rica; lo que a su vez conlleva a una ampliación hacia el sur del rango de distribución de la misma.

\section{Agradecimientos}

Un agradecimiento a IdeaWild.org por su apoyo con equipo de laboratorio; a Paul Hanson y a Monika Springer por permitir el acceso a la colección de enseñanza y a la Colección de Entomología Acuática del MZUCR respectivamente; a Robert W. Sites y Jean-Michel Maes por facilitar literatura. Esta publicación es una contribución al MZUCR.

\section{Literatura citada}

Champion, G.C. 1901. Insecta: Rhynchota (Hemiptera-Heteroptera), vol. II. In: F.D. Godman \& O. Salvin (eds.). Biologia Centrali-Americana, xvi +416 pp., plates $1-22$. London.

Herrera F. 2013. First report for the Neotropics of predation by the waterscorpion Ranatra (Hemiptera: Nepidae) on a member of Gerromorpha (Hemiptera). Cuadernos de Investigación UNED 5(1): 55-56.

Keffer S.L. 1997. Systematics of the New World Waterscorpion Genus Curicta Stål (Heteroptera: Nepidae). Journal of the New York Entomological Society 104(3-4), 1996: 117-215.

Keffer S.L. \& J.E. McPherson. 1993. Curictan copulation and waterscorpion higher classification (Heteroptera: Nepidae). Proceedings of the Entomological Society of Washington 95(1): 74-78.

Keffer S.L., S.J. Taylor \& J.E. McPherson. 1994. Laboratory rearing and descriptions of immature stages of Curicta scorpio (Heteroptera: Nepidae). Annals of the Entomological Society of America 87(1): 17-26.
Lago P.K. 2000. Mississippi record for Curicta scorpio (Hemiptera: Nepidae). Entomological News 111(4): 282.

Mazzucconi S., M.L. López-Ruf \& A.O. Bachmann. 2009a. HemipteraHeteroptera: Gerromorpha y Nepomorpha. Pp. 167-231. In: E. Domínguez \& H.R. Fernández (eds.). Macroinvertebrados bentónicos sudamericanos: Sistemática y biología. Fundación Miguel Lillo. Tucumán, Argentina. 656 p.

Mazzucconi, S. A., M. López-Ruf \& A.O. Bachmann. 2009b. Gerromorpha y Nepomorpha (Insecta: Heteroptera) del Parque Provincial Salto Encantado del Valle del Cuñá Pirú, Provincia de Misiones, Argentina. Lundiana 9 (1), 2008: 57-66.

Menke A.S. 1979. The Semiaquatic and Aquatic Hemiptera of California (Heteroptera: Hemiptera). Bulletin of the California Insect Survey 21: 1-166.

Montandon A.L. 1910. Nepidae. Notes et descriptions d'espèces nouvelles. Annales Musei Nationalis Hungarici 8: 163-171.

Sites R.W. \& J.T. Polhemus. 1994. Nepidae (Hemiptera) of the United States and Canada. Annals of the Entomological Society of America 87(1): 27-42.

Springer M. 1998. Genera of aquatic insects from Costa Rica, deposited at the Museo de Zoología, Universidad de Costa Rica. Revista de Biología Tropical 46(6): 137-141.

Springer M. 2004. (en línea). Lista de los géneros de insectos acuáticos depositados en el Museo de Zoología, Escuela de Biología, UCR. <http:// museo.biologia.ucr.ac.cr/Colecciones/GenerosInsectosAcuaticos. htm>. Acceso 09/12/13.

Štys P. \& A. Jansson. 1988. Check-list of recent family-group and genus names of Nepomorpha (Heteroptera) of the world. Acta Entomologica Fennica 50: 1-44.

Wiley G.O. 1922. Some notes on the biology of Curicta from Texas. Kansas University Science Bulletin 14(20): 507-511.

Wiley G.O. 1924. On the biology of Curicta drakei Hungerford (Heteroptera, Nepidae). Entomological News 192: 324-331. 